\title{
Antibiotic Sensitivity of Pathogens Causing Urinary Tract Infection
}

\author{
Taslima Akter ${ }^{1}$, Zakaria Mia ${ }^{2}$ and Masum Shahriar ${ }^{1}$ \\ ${ }^{1}$ Department of Pharmacy, Jahangirnagar University, Savar, Dhaka, Bangladesh \\ ${ }^{2}$ Department of Microbiology and Biotechnology, Jagannath University, Dhaka, Bangladesh
}

\begin{abstract}
Diagnosis of urinary tract infection (UTI) causing pathogens with their sensitivity to different antibiotics was performed with a total of 96 samples from both male $(n=31 ; 32.3 \%)$ and female $(n=65 ; 67.7 \%)$ of different age groups. Out of 96 urine samples, 55 (57.3\%) were found positive after culturing in MacConkey agar plates. The percent distribution of positive cases against collected samples was higher for female (67\%) than male (32\%). However, female and male at the reproductive age of $16-30$ years were more susceptible to UTI. A total of 55 bacterial isolates were identified by conventional methods and their antibiotic sensitivity was tested using MuellerHinton agar plates. The predominant isolates were Escherichia coli (34.5\%), Klebsiella sp. (18.2\%) and Staphylococci (20.0\%). The sensitivity pattern for most of the isolated organisms showed 50\% and/or higher sensitivity to imipenem, azithromycin and cephalexin, except Staphylococci (only $9.09 \%$ to azithromycin).
\end{abstract}

Keywords: Antibiotic sensitivity, bacterial pathogens, UTI

\section{Introduction}

Urinary tract infections (UTI) affect any part of the urinary tract and include mainly cystitis (bladder infection), pyelonephritis (kidney infection) and urethritis (urethra infection) showing tissue damage, burning, painful urination, urgency and increased urinary frequency, suprapubic pain, pain in renal angle, fever and other systemic manifestations but asymptomatic cases may also occur (Ramos et al., 1996; Calvin, 1994). Any anatomical barrier (prostatic hypertrophy, neurogenic disorder, tumor, stone, external catheter) to free flow of urine through the urinary tract contributes to the development of UTI and symptoms of UTI depend on the specific organ affected and type of organisms causing the disease (Baron and Finegold, 1990). Common invading pathogens of UTI are Escherichia coli, Klebsiella, Proteus, Pseudomonas, Streptococci, Staphylococci such as Staphylococcus saprophyticus, S. epidermidis, coagulase-negative staphylococci, Serratia, enterobacter and Gram-negative enteric bacteria and yeasts (Calvin, 1994; Atlas, 1995; Mark and Gordon, 1994). Usually UTIs are caused by single type of organism though polymicrobic infections are found in complicated cases with urinary stones and anatomical abnormality of urinary tract. Presence of $10^{5} \mathrm{cfu} / \mathrm{ml}$ in midstream urine is considered as significant number of bacteria for UTI (Kass, 1957) though this number is fewer for asymptomatic patients and patients using antimicrobials, consuming large amount of liquid, with urinary obstruction and pyelonephritis acquired from hematogenous spread. UTI is the most common nosocomial infection (Mark and Gordon, 1994) and remarkable differences on type of infecting organisms are evident in community acquired and hospitalized cases (Calvin, 1994). UTIs are more common in women than in men though male over 60 years with prostatic hypertrophy are the exceptions (Calvin, 1994; Jawetz et al., 1995).Women are more prone to UTIs than men because in females, the urethra is much shorter and closer to the anus than in males (Jawetz and Melnick et al., 1995) and they lack the bacteriostatic properties of prostatic secretions. The female genital tract is closely related to the bladder and this relationship makes the spread of diseases possible from one tract to the other (Epstein, 2010). Therapeutic decision should be based on accurate, up-todate anti-microbial susceptibility pattern. Interim data have been published from a European multi-centre survey that examined the prevalence and antimicrobial susceptibility of community acquired pathogens causing uncomplicated UTI in women (Kahlmeter, 2000). The duration of treatment for adult has received much attention. Traditionally, a course of 7-10 days has been advocated, still this is the recommendation for treating men. For women, though, there has been particular

Correspondence to: Masum Shahriar; Department of Pharmacy, Jahangirnagar University, Savar, Dhaka, Bangladesh;

E-mail address: masum_shahriar@yahoo.co.uk 
emphasis on the suitability of short-course regimens such as 3-days or even single-dose therapy. The consensus of an international expert working group was that 3-day regimens are as effective as longer regimens in the cases of trimethoprim and quinolones. $\beta$-Lactams have been inadequately investigated on this point but short courses are generally less effective than trimethoprim and quinolones and nitrofurantoin require further study before conclusion can be drawn (Warren et al., 1999).

Patients with pyelonephritis may be severely ill and if so, will require admission to hospital and initial treatment with a parenteral antibiotic. A first choice agent would be parenteral cefuroxime, gentamycin or ciprofloxacin. When the patient is improving, the route of administration may be switched to oral therapy, typically using a quinolone. Conventionally, treatment is continued for 10-14 days. Patients who are less severely ill at the outset may be treated with an oral antibiotic, and possibly with a shorter course of treatment. The safety of this approach has been demonstrated in a study of adult women with acute uncomplicated pyelonephritis (Talan et al., 2000). In hospital acquired pyelonephritis, there is a risk that the infecting organism may be resistant to the usual first-line drugs. In such cases it may be advisable to start a broad spectrum agent such as ceftazidime, ciprofloxacin or meropenem. In a study of women with catheter-associated infection, asymptomatic bacteriuria resolved spontaneously within 2 weeks in only 15 of 42 patients (Godfrey et al., 1991). However, those with persistent and symptomatic bacteriuria responded well to single-dose treatment.

Several different types of novel catheters with antiinfective properties have been developed with the aim of reducing the ability of bacteria to adhere to the material, which should lead to a decreased incidence of bacteriuria and symptomatic infection. Several studies of the effect of incorporating antibiotics such as rifampicin and minocycline (Darouchie et al., 1999) or silver based alloys (Newton et al., 2002) into the catheter have shown benefit. Although clearly more costly than standard catheters, economic evaluation shows silver alloy catheters to be cost effective when used in patients needing catheterization for several days (Plowman et al., 2001). The prevalence of asymptomatic bacteriuria of pregnancy is approximately $5 \%$, about a third of these women proceed to develop acute pyelonephritis, with its attendant consequences for the health of both mother and pregnancy. Furthermore, there is evidence that asymptomatic bacteriuria is associated with low birth weight, pre-maturity, hypertension and pre-eclampsia. For these reasons it is recommended that screening is carried out, preferably by culture of a properly taken MSU, which should be repeated if positive for confirmation (National Collaborating Centre for Women's and Children's Health 2003). Rigorous meta-analysis of published trials (Smaill, 2000) has shown that antibiotic treatment of bacteriuria in pregnancy is effective at clearing bacteriuria, reducing the incidence of pyelonephritis and reducing the risk of preterm delivery. The following measures may reduce the incidence of urinary tract infections. These may be appropriate for people, especially women, with recurrent infections (Mayon-White et al., 1988; Fisher et al., 1995; Talukder et al., 1987). Such as avoiding the delay of urination, cleaning the urethral opening with an antiseptic after intercourse, fruit juice (cranberry, blueberries) containing tannins can decrease the incidence of UTI, tannins prevent the adherence of certain pathogens ( $E$. coli) to the epithelium of the urinary bladder, intravaginal application of topical estrogen cream and long courses of low-dose antibiotics taken at night can prevent recurrent cystitis, breast feeding can reduce the risk of UTIs in infants. In children, recurrence of UTI is a common and the complications potentially hazardous, so many clinicians recommended anti-microbial prophylaxis following documented infection. The evidence in favor of this practice is not strong (Le Saux et al., 2000), and although it has been shown to reduce the incidence of UTI, it has not been shown to reduce the incidence of renal complications. Indiscriminate use of antibiotics and other related factors are responsible for the evolution of many drug resistant microbial strains causing infections including UTI. Moreover, the ever changing pattern of sensitivity indicates the importance of continuous investigation for updating relevant data for intended use as guidelines for appropriate treatment by the physicians. And hence the present study was undertaken to find out the most frequent microbial cause of UTI and the patterns of their sensitivity to different antibiotics in order to facilitate better treatment and management of UTIs. 


\section{Materials and Methods}

The study was performed at Gonoshasthaya Vaccine Research and Diagnostic Laboratory (GVRL), Department of Microbiology, Gono Bishwabidyalay (Gono University) Savar, Dhaka. The culture media used were MacConkey agar (Oxoid Ltd., England), Tryptic soy agar (Becton Dickinson and Company, USA) and Mueller Hinton agar (Sanofi Diagnostics, Pasteur, France). Sheep blood was collected from animal house of GVRL, and other chemical ingredients were of appropriate analytical and commercial standards.

Sample: Midstream urine samples were collected from the hospital patients suspected for UTI by paramedic staff and from the referred patients of UTIs who visited outdoor of Gonoshasthaya Hospital, Savar, Dhaka. Written consent was obtained from all patients.

Isolation of bacteria: Qualitative microbiological analysis was performed with all the samples. MacConkey agar and 5\% sheep blood-agar support growth of most Gram-negative bacilli and staphylococci (Baron and Finegold, 1990) and accordingly, inoculation on these media was done with pre-calibrated platinum loop to deliver a measured quantity $(1.0 \mu \mathrm{l})$ of urine sample. The inoculation at $37^{\circ} \mathrm{C}$ for 48 hours and CFU count of $10^{5} / \mathrm{ml}$ of urine were considered positive for UTI. Identification of organisms was done by conventional methods through culturing of samples followed by biochemical tests (Monica, 1984) including their distinct colony characteristics.

Sensitivity testing: Antimicrobial sensitivity was performed by disc diffusion method on Mueller-Hinton agar plates for organisms other than streptococci which needed blood agar plates. Antibiotic discs were purchased from commercial suppliers of the manufacturers Becton Dickinson and Company, USA; Oxoid Ltd. UK; Mast Diagnostics, UK. All bacteria were assayed against the following antimicrobial agents: Imipenem (10 $\mu \mathrm{g})$, Aztreonam (30 $\mu \mathrm{g})$, Ciprofloxacin (5 $\mu \mathrm{g})$, Tetracycline (30 $\mu \mathrm{g})$, Amoxicillin $(30 \mu \mathrm{g})$, Cotrimoxazole $(25 \mu \mathrm{g})$, Gentamycin $(120 \mu \mathrm{g})$, Azithromycin (15 $\mu \mathrm{g})$, Nalidixic

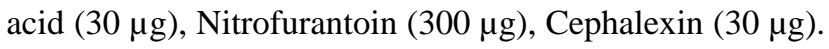
Strains were defined as susceptible, intermediate-resistant or resistant according to CLSI recommendations (CLSI, 2006).

\section{Results and Discussion}

UTI in females is among the most frequent infections in the community. This study has assessed the epidemiology and susceptibility patterns of common uropathogens to standard antimicrobials used in the treatment of uncomplicated lower UTI. A total of 96 clinical urine samples were collected of which 31 (32.3\%) were from male and 65 (67.7\%) were from female. Out of the collected 96 urine samples, 55 samples (57.3\%) were found positive with one organism for each after culturing. For both suspected patients for UTI attending the hospital and positive findings of diagnosis are higher for female than that of male and this was similar to the established data to date. Prevalence of high percentage of positive samples also indicates the low level sanitations practices among the attendees in hospital with UTI complications who are mostly from poor rural community. UTIs account for $67.5 \%$ (Calvin, 1994) as higher and 52.6\% (Zakaria et al., 2002) almost similar to that of present study 55 among 96 samples (57.3\%). Total culture positive by $57.3 \%$ of the samples shows similarity to the study of Rahman et al. (1990) in non-diabetic patients. The findings clearly show that both female and male patients of reproductive age (16 to 30 years) were infected in higher percentage by UTI $(27.8 \%$ of male and $45.9 \%$ of female) with similar reports (Calvin, 1994; Rahman, 1990). In contrast, Zakaria et al. (2002) reported that the incidences at reproductive age (16-35 yrs) are $31.25 \%$ for male and 69.96 for female. With other reasons, the high occurrence of UTI at this age might be due to their initial exposure to sex, and independent movement in environment without enough hygiene practices at this age. Moreover, the higher prevalence of UTI in female (67.3\%) compared to that of male (32.7\%) (Table 1) resembles with that reported earlier (72.5\% for female and $27.5 \%$ for male (Zakaria et al. (2002).

Table 1. Samples with UTI causing bacteria in different sex groups.

\begin{tabular}{lcc}
\hline Sex & No. (\%) of samples & No. (\%) positive cases \\
\hline Male & $31(32.3)$ & $18(32.7)$ \\
Female & $65(67.7)$ & $37(67.3)$ \\
Total & $96(100)$ & $55(57.3)$
\end{tabular}

From 55 positive samples, a total of 55 isolates of different organisms were isolated, where no sample showed polymicrobic UTI that is reported by early 
workers. E. coli is a common cause of UTI in other studies followed by next leading microbes as Klebsiella, Pseudomonas, Staphylococci, and also in the present study E. coli showed higher prevalence than other organisms i.e. E. coli (34.5\%), Klebsiella spp. (18.2\%), Staphylococci (20.0\%), Pseudomonas (9.1\%), and various Gramnegative Bacilli (18.2\%) (Table-3). Baron (1990) also reported that E. coli, Klebsiella, Pseudomonas and Staphylococci were the most common organisms associated with UTI for both community acquired and hospitalized cases.

Table 2. Distribution of positive cases of UTI in different age groups.

\begin{tabular}{ccc}
\hline \multirow{2}{*}{ Age group } & \multicolumn{2}{c}{ Positive for UTI } \\
\cline { 2 - 3 } & Male number (\%) & Female number (\%) \\
\hline $0-15$ & $5(27.8 \%)$ & $06(16.2 \%)$ \\
$16-30$ & $5(27.8 \%)$ & $17(45.9 \%)$ \\
$31-45$ & $2(11.1 \%)$ & $09(24.3 \%)$ \\
$46-60$ & $3(16.7 \%)$ & $01(02.7 \%)$ \\
$>60$ & $3(16.7 \%)$ & $04(10.8 \%)$ \\
Total & $18(100 \%)$ & $37(100 \%)$ \\
\hline
\end{tabular}

Table 3. Bacterial isolates from urine samples of patients with UTI.

\begin{tabular}{|c|c|c|}
\hline Organisms & Number of isolates & Percent \\
\hline E. coli & 19 & $34.5 \%$ \\
\hline Klebsiella sp. & 10 & $18.2 \%$ \\
\hline Staphylococci & 11 & $20.0 \%$ \\
\hline Pseudomonas sp. & 05 & $9.1 \%$ \\
\hline Other Gram-negative Bacilli & 10 & $18.2 \%$ \\
\hline Total & 55 & $100 \%$ \\
\hline
\end{tabular}

The antibiotic sensitivity of the isolates obtained from the UTI suspected subjects showed the sensitivity pattern presented in table-4. From the data, it is clear that most of the isolated organisms showed $50 \%$ or higher sensitivity to imipenem, azithromycin and cephalexin separately, except Staphylococci (only 9.09\% to azithromycin). Table 4 shows that all the isolates highly sensitive to imipenem (IMP) even up to $100 \%$ by staphylococci. E. coli showed less than $50 \%$ (10.5-47.7\%) sensitivity to 8 antibiotics out of 11 used. And it was (20-40\%) for Klebsiella to 5 antibiotics out of 11 .

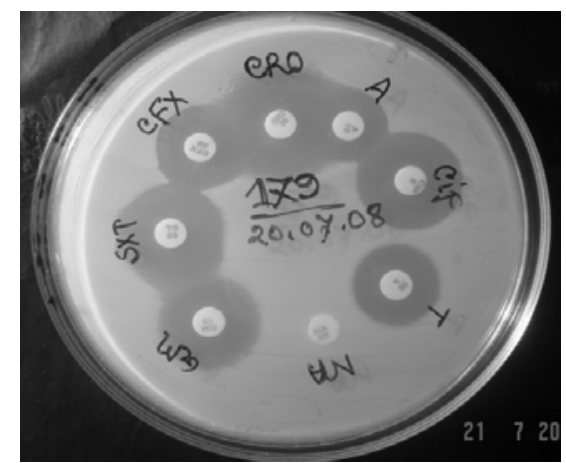

Figure 1. A sensitivity plate (Mueller-Hinton agar) showing the resistance and sensitivity against different antimicrobial agents.

Pseudomonas was completely resistant to TE, AMP, NI, CFX, and highly resistant to AZT, CIP, GM, NA by 20\%. Higher resistance pattern of Pseudomonas as described here was not observed earlier. Increasing resistance to different drugs by the most common pathogens causing UTI is alarming and indicates the treatment failure by these antibiotics. However, IMP, ATH and CFX seemed to be more effective with higher sensitivity.

Table 4. Bacterial isolates with their sensitivity to various antibiotics.

\begin{tabular}{|c|c|c|c|c|c|c|c|c|c|c|c|c|}
\hline \multirow[t]{2}{*}{ Type of isolates } & \multirow{2}{*}{$\begin{array}{l}\text { No. of } \\
\text { isolates }\end{array}$} & \multicolumn{11}{|c|}{ Sensitivity to different antibiotics (\%) } \\
\hline & & IMP & AZT & CIP & TE & AMP & SXT & GM & ATH & NA & NI & CFX \\
\hline E. coli & 19 & 95 & 47.4 & 47.4 & 21.1 & 21.1 & 10.5 & 26.3 & 84.2 & 31.6 & 26.3 & 57.9 \\
\hline Klebsiella sp. & 10 & 88 & 70 & 60 & 20 & 40 & 30 & 60.0 & 60.0 & 40.0 & 20.0 & 50.0 \\
\hline Staphylococci & 11 & 100 & 45.5 & 36.4 & 00 & 54.5 & 63.6 & 72.7 & 9.09 & 18.2 & 9.09 & 63.6 \\
\hline Pseudomonas sp. & 5 & 60 & 20.0 & 20.0 & 00 & 00 & 40.0 & 20.0 & 60.0 & 20.0 & 00 & 00 \\
\hline $\begin{array}{l}\text { Other Gram- } \\
\text { negative Bacilli }\end{array}$ & 10 & 80 & 20.0 & 60.0 & 60 & 30.0 & 40.0 & 30.0 & 60.0 & 50.0 & 00 & 30.0 \\
\hline
\end{tabular}

Different antibiotic discs used in sensitivity tests (quantity/disc) are: IMP: Imipenem (10 $\mu$ g), AZT: Aztreonam(30 $\mu$ g), CIP: Ciprofloxacin (5 $\mu \mathrm{g}), \mathrm{TE}$ : Tetracycline $(30 \mu \mathrm{g})$, AMP: Amoxicillin $(30 \mu \mathrm{g})$, SXT: Cotrimoxazole $(25 \mu \mathrm{g})$, GM for Gentamycin $(120 \mu \mathrm{g})$, ATH: Azithromycin (15 $\mu \mathrm{g})$, NA: Nalidixic acid $(30 \mu \mathrm{g})$, NI: Nitrofurantoin $(300 \mu \mathrm{g})$, CFX: Cephalexin $(30 \mu \mathrm{g})$.

A comparative study including Rahman et al. (1990),Talukder et al. (1987), Zakaria et al. (2002) and Islam et al. (2009) is presented in Table-5. Here it is evident that the predominant organisms are becoming dramatically resistant to most antibiotics in course of time that might be due to indiscriminate use of antibiotics by UTI patients and incomplete course of antibiotic use by the patients showing an ultimate fate of endangering the mankind in treatment perspective. 
Table 5. Sensitivity of commonly UTI causing organisms at different time.

\begin{tabular}{|c|c|c|c|c|c|c|}
\hline \multirow[t]{2}{*}{ Organisms } & \multirow[t]{2}{*}{ Antibiotics } & \multicolumn{5}{|c|}{ Sensitivity (\%) } \\
\hline & & Talukder (1987) & Rahman (1990) & Zakaria (2002) & Islam (2009) & Present study \\
\hline \multirow[t]{4}{*}{ E. coli } & Cephalexin & - & 67.0 & 38.6 & - & 57.9 \\
\hline & Nalidixic acid & 85 & 80.0 & 34.7 & 67.7 & 31.6 \\
\hline & Nitrofurantoin & 90 & 81.5 & 56.4 & 96.8 & 26.3 \\
\hline & Gentamycin & 100 & 78.0 & 58.4 & 51.6 & 26.3 \\
\hline \multirow{4}{*}{$\begin{array}{l}\text { Klebsiella } \\
\text { spp. }\end{array}$} & Cephalexin & - & 70.0 & 51.2 & - & 50.0 \\
\hline & Nalidixic acid & - & 64.0 & 56.0 & - & 40.0 \\
\hline & Nitrofurantoin & - & 76.0 & 65.6 & - & 20.0 \\
\hline & Gentamycin & - & 70.0 & 79.2 & - & 60.0 \\
\hline
\end{tabular}

The present findings resembles with the established truth to date that E. coli and Klebsiella are the leading uropathogens causing infections mostly at reproductive age and females are more sufferers of UTI. Exceptionally, it shows that Staphylococci are $9.09 \%$ sensitive to azithromycin, a broad-spectrum antibiotic, though it was $100 \%$ sensitive to imipenem. From the comparative data, it is also clear that the bacteria representing as the predominant causes of UTI are becoming resistant to the most commonly used antibiotics for UTI treatment. These data provide information on antimicrobial resistance pattern among pathogens currently causing UTI in Savar area. Since the resistance pattern of pathogens are ever changing and continuous, dealing with a limited number of samples the present study emphasizes on the necessity of a broad-based study that can reflect the authentic data and reliable information as treatment guidelines for UTIs.

\section{References}

Atlas, R.M. 1995. Principles of Microbiology. Missouri, St. Louis, USA: CV Mosby Company, p. 491.

Baron, E.J. and Finegold, S.M. 1990. Bailey and Scott's Diagnostic Microbiology, $8^{\text {th }}$ Ed. Baltimore, Philadelphia, USA; The CV Mosby Company. p. 253-261.

Calvin, M.K. 1994. Urinary tract infections in females. Clin. Infec. Dis. 18, 1-12 .

Clinical and Laboratory Standards Institute. 2006. Methods for dilution antimicrobial susceptibility tests for bacteria that grow aerobically. 7th ed. Approved standard M7A7.Wayne, PA: CLSI.

Darouchie, R.O., Smith, J.A. Jr. and Hanna, H. 1999 Efficacy of antimicrobial impregnated bladder catheters in reducing catheter associated bacteriuria: a prospective, randomized, multicenter clinical trial. Urology 54, 976-981
Epstein, J.I. 2010. The lower urinary tract and male genital system, chapter 21 in "Robbins and Cotran Pathologic basis of disease". $8^{\text {th }}$ ed. by Kumar V, Abbas A.K., Fausto N, Aster J.C., Elsevier, Haryana, India.

Fisher, J.F., Newman, C.L. and Shobel, J.D. 1995. Yeast in the urine: Solutions for a budding problem. Clin. Infec. Dis. 20, 183-189.

Godfrey, K.M., Lindsay, E.N. and Ronald, A.R. 1991. How long catheter-acquired urinary tract infection in women should be treated? Annals Inter. Med. 114, 713-719.

Islam M.A., Saha R.R. and Zakaria M.M. 2009. Incidence and antibiotic resistance in Escherichia coli isolated from clinical samples at Gonoshasthaya Hospital, Savar. Bangladesh J. Med. Sci. 15, 141-142.

Jawetz, E. and Melnick, 1995. Clinical correlations: urinary tract in Medical Microbiology, $20^{\text {th }}$ Ed. London, UK, Prentice Hall Intl Inc. p. 634.

Kahlmeter, G. 2000. The ECOSEN project: a prospective, multinational, multicentre epidemiological survey of the prevalence and antimicrobial susceptibility of urinary tract pathogens. Interim report. J. Antimicrob. Chemother. (Suppl. SI), 15-22.

Kass, E.H. 1957. Bacteriuria and the diagnosis of infections of the urinary tract: with observations on the use of methionine as a urinary antiseptic. Arch. Intern. Med. 100, 709-714.

LeSaux, N., Pham, B. and Mother, D. 2000. Evaluating the benefits of antimicrobial prophylaxis to prevent urinary tract infections in children: a systematic review. Canadian Med. Assoc. J. 163, 523-529.

Mark, E.R. and Gordon, L.A. 1994. Coagulase-negative staphylococci: pathogens associated with medical progress. Clin. Infec. Dis. 19, 231245.

Monica, C. 1984. Medical laboratory manual for tropical countries. Vol. II, first Ed. 14 Bevills Close, Doddington, Cambridgeshire, England: Tropical Health Technology, 146. 
Mayon-White, R.T. and Ducel, G. 1988. An international survey of the prevalence of hospital acquired infection. J. Hosp. Infec. 11(suppl A), 43-48.

National Collaborating Centre for Women's and Children's Health, 2003. Antenatal care: routine care for the healthy pregnant woman. Royal College of Obstetricians and Gynaecologists Press. London. pp. 79-81.

Newton, T., Still, J.M. and Law, E. 2002. A comparison of the effect of early insertion of standard latex and silverimpregnated latex Foley catheters on urinary tract infections in burn patients. Infect. Control Hosp. Epidemiol. 23, 217218

Plowman, R., Graves, N. and Esquivel, J. 2001. An economic model to assess the cost and benefits of the routine use of silver alloy coated urinary catheters to reduce the risk of urinary tract infections in catheterized patients. J. Hosp. Infect. 48, 33-42.

Rahman, T. and Haq, F. 1990. Urinary tract infecton (UTI) in diabetic and non-diabetic patients-A comparaive bacteriological study. Bangladesh Renal J. 9, 8-12.

Ramos, J.M. and Aguado, J.M. 1996. Clinical spectrum of UTI due to non-typhoidal Salmonella sp. Clin. Infec. Dis. 23, 388-390.
Smaill, F. 2000. Antibiotics for asymptomatic bacteriuria in pregnancy (Cochrane Review). Cochrane Library, Issue 4. Update Software, Oxford

Talan, D.A., Stamm, W.E. and Hooton, T.M. 2000. Comparision of ciprofloxacin (7 days) and trimethoprimesulfamethoxazole (14 days) for acute uncomplicated pyelonephritis in women: a randomized trial, J. American Med. Assoc. 283, 1583-1590.

Talukder, M.A.S., Bakheshwain, S.M. and Altasan, F. 1987. Urinary tract infection in children at the Armed Forces Hospital, Riyadh, Saudi Arabia-A pilot study. Bangladesh J. Child. Health. 11, 55-58.

Warren, J.W., Abrutyn, E. and Herbal, J.R 1999 Guidelines for antimicrobial treatment of uncomplicated acute bacterial cystitis and acute pyelonephritis in women. Clin. Infect. Dis. 29, 745-758.

Zakaria, M.M., Talukder, A.S. and Chowdhury, E.K. 2002. Prevalence and Drug Sensitivity of microorganisms in patients with Urinary tract infection attending a semi-rural hospital in Bangladesh. Bangladesh J. Med. Sci. 8, 111-114. 